\title{
Increased Risk of Depression in Patients with Cholesteatoma: A 3-year Nationwide Population-Based Retrospective Cohort Study
}

\author{
Chin-Lung Kuo, MD, PhD ${ }^{1,4,5}$; Wei-Pin Chang, $\mathrm{PhD}^{2 *}$; Nancy Hsao Yen Chang, $\mathrm{BSN}^{3}$; An-Suey Shiao, $\mathrm{MD}^{4,5}$; \\ Chiang-Feng Lien, $\mathrm{MD}^{4,5}$
}

${ }^{1}$ Department of Otolaryngology, Taoyuan Armed Forces General Hospital, Taoyuan, Taiwan

${ }^{2}$ School of Health Care Administration, Taipei Medical University, Taipei, Taiwan

${ }^{3}$ Institute for Cellular and Molecular Biology, University of Texas at Austin, Austin, Texas, USA

${ }^{4}$ Department of Otolaryngology-Head and Neck Surgery, Taipei Veterans General Hospital, Taipei, Taiwan

${ }^{5}$ Department of Otolaryngology, National Yang-Ming University School of Medicine, Taipei, Taiwan

Abstract

Objective: Cholesteatoma is a locally invasive condition; however, a comorbidity of depression could be a risk factor of systemic morbidity and mortality. A relationship between cholesteatoma and depression has been observed in clinical practice; however this link has not been firmly established in the literature. This study sought to estimate the risk of developing depressive disorder (DD) following diagnosis with cholesteatoma. Methods: In the study, we analyzed data from the Longitudinal Health Insurance Database of Taiwan. A total of 1820 patients newly diagnosed with cholesteatoma between 1997 and 2007 were included with a comparison cohort of 9100 matched non-cholesteatoma enrollees. Each patient was followed for 3 years to identify the subsequent development of DD. Cox proportional hazard regression analysis was performed to compute adjusted 3-year hazard ratios.

Results: Of the 10,920 patients in the sample, 53 individuals (2.9\%) from the cholesteatoma cohort, and 149 (1.6\%) from the comparison cohort were subsequently diagnosed with DD during the 3-year follow-up. The incidence of DD per thousand person-years was approximately twice as high among patients with cholesteatoma (9.86) as among those without cholesteatoma (5.50). After adjusting for potential confounders, patients with cholesteatoma were 1.69 times $(95 \% \mathrm{Cl}=1.21-2.36, P=0.002)$ more likely to suffer from DD within 3 years compared to those without cholesteatoma.

Conclusion: This is the first study to demonstrate a prospective link between cholesteatoma and subsequent DD within a three-year follow-up. Routine psychiatric screening among cholesteatoma patients is essential for the early detection and timely treatment of depression.

\section{Introduction}

Cholesteatoma is a well-demarcated lesion which develops from an abnormal growth of keratinizing squamous epithelium in the temporal bone [1]. The annual incidence of cholesteatoma is between 9.2 and 12.6 cases per 100,000 adults, with predominance in Caucasian populations [2]. Cholesteatoma is histologically benign but behaviorally malignant [3]. The dysregulation of cell growth control with local invasive characteristics is the major mechanism underlying widespread structural destruction in the middle ear cleft. The goal of treatment is typically complete disease eradication. Given the fatal capacity of intracranial complications, cholesteatoma remains a risk factor of morbidity and mortality in areas where access to appropriate medical care may be limited [4]. Patients

*Correspondence: Wei-Pin Chang, PhD

School of Health Care Administration, Taipei Medical University, Taipei, Taiwan. Email: wpchang@tmu.edu.tw

Received: Aug. 16, 2017; Accepted: Sep. 16, 2017; Published: Oct. 03, 2017

Archives of Otorhinolaryngology-Head \& Neck Surgery. 2017;1(3):1

DOI: 10.24983/scitemed.aohns.2017.00038

Copyright (c) 2017 The Author(s). This is an open-access article distributed under the terms of the Creative Commons Attribution 4.0 International License (CC-BY). with cholesteatoma are susceptible to frequent recurrence of foul-smelling otorrhea, hearing impairment, and tinnitus [4]. Depression commonly occurs in the context of medical conditions, with a prolonged impact on patients' quality of life [5]. Thus, it is reasonable to assume that the psychological burden associated with cholesteatoma could cause a patient to become socially withdrawn and potentially lead to the development of depressive disorder (DD).

Investigating the potential link between physical and mental illnesses may provide clues to enable the early detection of DD. Medical comorbidity with DD is a major public health concern [6]. Clinicians dealing with cholesteatoma have tended to place primary focus on hearing outcomes and disease control, such that the relationship between cholesteatoma and depression remains unclear. However, it is worth noting that one previous case report did address this topic [7].

In this current study, we sought evidence to support our hypothesis that patients with cholesteatoma may face an elevated risk of developing DD. To the best of our knowledge, this is the first population-based study to investigate a link between depression and cholesteatoma.

\section{Methods}

\section{Ethics statements}

The Institutional Review Board of Taipei Veterans General Hospital exempted this study from review because it used existing, de-identified public use data 
(VGHIRB No. 2015-04-002AC).

\section{Data source}

The National Health Insurance (NHI) program was implemented in 1995 and currently covers $99 \%$ of the Taiwanese population [8]. Claims data are maintained in the National Health Insurance Research Database (NHIRD), which is managed and audited by the National Health Research Institutes (NHRI). The NHIRD includes data related to demographics, clinical visits, diagnostic codes, prescriptions, and expenditures. To validate the completeness and accuracy of the claims data, the NHRI conducts random patient interviews, annually reviews medical records, and employs random sampling of claims from every hospital. Hundreds of researchers have previously employed $\mathrm{NHI}$ data in peer-reviewed research $[8,9]$.

This retrospective study used a subset of the NHIRD, the Longitudinal Health Insurance Database (LHID2005), which enables researchers to follow-up on all medical service utilization by 1 million enrollees randomly selected from the NHIRD in 2005. The NHRI has stated that there are no statistically significant differences related to age, sex, or health-care costs between the LHID 2005 and the NHIRD [8].

We examined ambulatory and inpatient care data for patients in the LHID2005 from 1997 to 2007. To ensure privacy, the original identification number of each patient in the LHID2005 is encrypted before it is linked to personal health-care information.

\section{Participants}

A study group and a comparison group were assembled for a retrospective cohort examination of the relationship between cholesteatoma and depression. Patients newly diagnosed with cholesteatoma between January 1, 1997 and December 31, 2007 were identified according to diagnostic criteria from the International Classification of Diseases, Ninth Revision, Clinical Modification (ICD-9-CM code 385.3, 385.30, 385.31, 385.32, 385.33). The date of the first diagnosis with cholesteatoma was assigned as the index date for each patient in the study. To ensure the accuracy of data, only patients with $\geq 2$ ambulatory visits or $\geq 1$ inpatient visits for cholesteatoma were included in the case cohort. Five patients without cholesteatoma were randomly matched to each patient in the cholesteatoma cohort based on age, sex, and index year. Patients with a history of depression (ICD-9-CM code 296.2, 296.3, 300.4, 311) were excluded from this study, and hypertension, hyperlipidemia, diabetes, and obesity were treated as potential confounders for the risk of depression. Finally, we adopted similar criteria for depression as we did for cholesteatoma, including in the cohort only cases with $\geq 2$ ambulatory visits or $\geq 1$ inpatient visit. In addition, depression diagnostic codes had to have been assigned by a psychiatrist.

\section{Level of urbanization}

We matched the levels of urbanization to avoid potential confounders, such as unmeasured neighborhood socioeconomic characteristics between the study cohort and comparison cohort. In the LHID 2005, the 359 towns/cities in Taiwan are stratified into 8 urbanization categories according to criteria established by the NHRI, with 1 indicating the highest level of urbanization and 8 indicating the lowest. The criteria include population density (persons per $\mathrm{km}^{2}$ ), the percentage of people with a collegelevel education or higher, the percentage of people aged 65 years or older the percentage of agricultural workers in the local population, and the number of physicians per 100000 people. However, the number of cholesteatoma cases in levels 4, 5, 6, 7, and 8 were low; therefore, these levels were combined into a single urbanization group, level 4.

\section{Statistical analysis}

Pearson chi-square tests were performed to examine differences in categorical data between cholesteatoma and comparison cohorts, including level of urbanization, monthly income, geographic region, and potential confounders (i.e. hypertension, hyperlipidemia, diabetes, or obesity). Parametric continuous data for the two cohorts, including follow-up time, were compared using the Students $t$-test.

DD-free survival was calculated for all patients diagnosed with depression between the date of the first hospitalization or ambulatory visit for cholesteatoma and the end of the study period (December 31, 2010) or death, whichever came first. We calculated the three-year DD-free survival rates using the Kaplan-Meier method. We also applied the log-rank test to examine differences in DD-free survival rates between cohorts. Cox proportional-hazard regression analysis stratified by sex, age group, index year, and relative confounders was used to examine the risk of DD in cholesteatoma and comparison cohorts during the 3-year follow-up period. We also examined the effects of sex, age, hypertension, hyperlipidemia, diabetes, and obesity on the relationship between cholesteatoma and depression events. For the stratified Cox regression analysis, patients were divided into four categories: 0-19, 20-39, 40-59, and $\geq 60$ years. Hazard ratios (HRs) and $95 \%$ confidence intervals ( $\mathrm{Cls}$ ) were calculated to represent the risk of depression in the cohorts before and after stratification based on age or sex. Comparison results had to have a 2 sided $P$ value of $<0.05$ to be considered statistically significant.

\section{Results}

A total of 1820 and 9100 patients were included in the cholesteatoma and comparison cohorts, respectively. Sociodemographic and comorbidity data for both the cholesteatoma and comparison cohorts are listed in Table 1. Cholesteatoma patients presented higher rates of hyperlipidemia $(P=0.028)$ and diabetes $(P=0.002)$ than did the comparison cohort. Cholesteatoma patients were also more likely to have a lower monthly income $(P<0.001)$ or reside in central Taiwan $(P<0.001)$. No statistically significant differences in hypertension $(P=0.142)$ or obesity $(P=0.195)$

Table 1. Demographic characteristics for the selected patients, stratified by presence/absence of cholesteatoma from 1997 to 2007 ( $n=10,920)$

\begin{tabular}{|c|c|c|c|c|c|}
\hline \multirow[t]{2}{*}{ Variable } & \multicolumn{2}{|c|}{$\begin{array}{l}\text { Patients with } \\
\text { cholesteatoma } \\
(n=1820)\end{array}$} & \multicolumn{2}{|c|}{$\begin{array}{l}\text { Patients without } \\
\text { cholesteatoma } \\
(n=9100)\end{array}$} & \multirow[t]{2}{*}{$P$ value } \\
\hline & $\mathrm{n}$ & $\%$ & $\mathrm{n}$ & $\%$ & \\
\hline Gender & & & & & 1 \\
\hline Male & 882 & 48.5 & 4410 & 48.5 & \\
\hline Female & 938 & 51.5 & 4690 & 51.5 & \\
\hline Age (years) & & & & & 1 \\
\hline $0-19$ & 117 & 6.4 & 585 & 6.4 & \\
\hline $20-39$ & 380 & 20.9 & 1900 & 20.9 & \\
\hline $40-59$ & 824 & 45.3 & 4120 & 45.3 & \\
\hline $60-$ & 499 & 27.4 & 2495 & 27.4 & \\
\hline Follow-up, year, mean & & & & & 0.003 \\
\hline & 2.95 & 0.32 & 2.98 & 0.22 & \\
\hline Urbanization level & & & & & $<0.001$ \\
\hline 1 (most urbanized) & 546 & 30.0 & 2964 & 32.6 & \\
\hline 2 & 558 & 30.7 & 2497 & 27.4 & \\
\hline 3 & 380 & 20.9 & 1515 & 16.6 & \\
\hline 4 (least urbanized) & 336 & 18.5 & 2124 & 23.3 & \\
\hline Monthly income & & & & & $<0.001$ \\
\hline 0 & 534 & 29.3 & 2373 & 26.1 & \\
\hline NT\$ 1-15840 & 244 & 13.4 & 1015 & 11.2 & \\
\hline NT\$ $15841-25000$ & 687 & 37.7 & 3686 & 40.5 & \\
\hline NT\$ $\geq 25001$ & 355 & 19.5 & 2026 & 22.3 & \\
\hline Geographic region & & & & & $<0.001$ \\
\hline North & 522 & 28.7 & 4248 & 46.7 & \\
\hline Central & 962 & 52.9 & 2284 & 25.1 & \\
\hline South & 284 & 15.6 & 2137 & 23.5 & \\
\hline Eastern & 52 & 2.9 & 431 & 4.7 & \\
\hline Hypertension & & & & & 0.142 \\
\hline Yes & 853 & 46.9 & 4094 & 45.0 & \\
\hline No & 967 & 53.1 & 5006 & 55.0 & \\
\hline Hyperlipidemia & & & & & 0.028 \\
\hline Yes & 750 & 41.2 & 3499 & 38.5 & \\
\hline No & 1070 & 58.8 & 5601 & 61.5 & \\
\hline Diabetes & & & & & 0.002 \\
\hline Yes & 548 & 30.1 & 2414 & 26.5 & \\
\hline No & 1272 & 69.9 & 6686 & 73.5 & \\
\hline Obesity & & & & & 0.195 \\
\hline Yes & 39 & 2.1 & 155 & 1.7 & \\
\hline No & 1781 & 97.9 & 8945 & 98.3 & \\
\hline
\end{tabular}


Table 2. Hazard ratios (HRs) of Depressive disorders among cholesteatoma patients during the 3-year follow-up period from the index ambulatory visits or inpatient care from 1997 to 2007

\begin{tabular}{|c|c|c|c|c|c|c|c|}
\hline \multirow[t]{2}{*}{ Development of depressive disorders } & \multicolumn{2}{|c|}{ Total } & \multicolumn{2}{|c|}{ Patients with cholesteatoma } & \multicolumn{2}{|c|}{ Patients without cholesteatoma } & \multirow[t]{2}{*}{$P$ value } \\
\hline & $\mathrm{n}$ & $\%$ & $\mathrm{n}$ & $\%$ & $\mathrm{n}$ & $\%$ & \\
\hline \multicolumn{8}{|l|}{ 3-year follow-up period } \\
\hline Yes & 202 & 1.8 & 53 & 2.9 & 149 & 1.6 & \\
\hline No & 10718 & 98.2 & 1767 & 97.1 & 8951 & 98.4 & \\
\hline Crude HR (95\% Cl) & & & & $1.79(1.31-2.45)^{\star \star \star}$ & 1 & & $<0.001$ \\
\hline Adjusted HR (95\% Cl) & & & & $1.69(1.21-2.36)^{\star \star}$ & 1 & & 0.002 \\
\hline
\end{tabular}

Total sample number $=10920$; both crude and adjusted HRs were calculated by Cox proportional hazard regressions, and stratified by age and sex. Adjustments were made for patients' sex, age, urbanization level, monthly income, geographic region, hyperlipidemia, and diabetes. *Indicates $p<0.05 ; * *$ Indicates $p<0.01$; ***Indicates $p<0.001$

were observed. During the 3-year follow-up, 53 (2.9\%) of the cholesteatoma patients and $149(1.6 \%)$ of the patients in the comparison group developed DD. Cox regression analysis revealed that the crude HR of DD was 1.79 times higher $(95 \% \mathrm{Cl}=1.31-2.45 ; P<0.001)$ among cholesteatoma patients than among the comparison cohort. The risk of DD remained significant even after adjusting for potential confounders (adjusted HR: 1.69, 95\% Cl: 1.21-2.36; $P=0.002$; Table 2), and cholesteatoma patients presented a significantly lower 3 -year DD-free survival rate $(P<0.001$; Figure 1).

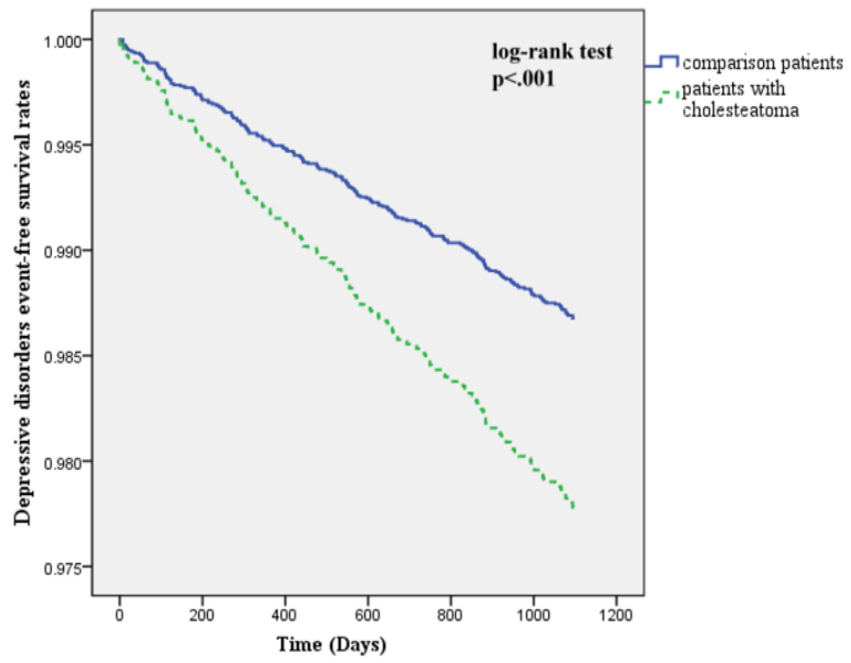

Figure 1. Depression-free survival rates in the cholesteatoma and control groups from 1997 to 2007

To investigate whether cholesteatoma is a time-dependent risk factor for $\mathrm{DD}$, patients were divided into three subgroups according to the duration of follow-up (i.e. 1, 2, or 3 years). In all three follow-up periods, the risk of developing DD remained significantly higher in the cholesteatoma group than in the comparison cohort both before and after adjusting for potential confounders (Table 3).

In the three-year follow-up period, the overall DD incidence density was higher among patients with cholesteatoma ( 9.86 per 1000 personyears) than in the comparison cohort (5.50 per 1000 person-years) (Table 4). Gender and age stratified analysis of incidence revealed that women had a higher incidence of DD than men (11.60 vs. 8.02 per 1000 personyears) and that patients aged over 60 years had a higher incidence of DD (14.33 per 1000 person-years) than all other age groups (Table 4).

Cox regression analysis revealed that the difference in the risk of DD between male patients and the male control group (adjusted HR 1.78, $95 \% \mathrm{Cl}=1.02-3.10 ; P<0.05$ ) was more pronounced than was the difference in risk of DD between female patients and the female control group (adjusted HR 1.63, 95\% Cl =1.07-2.48; $P<0.05$ ) (Table 4). In addition, the difference in the risk of DD among patients aged 20 to 39 years and the control group of patients of the same age (adjusted HR 4.05, 95\% Cl $=1.60-10.24 ; P<0.01$ ) was higher than the difference in the risk of DD between patients and controls in other age groups. With regard to follow-up period, it was found that patients with cholesteatoma had the highest risk of developing DD within one year of diagnosis (adjusted HR 1.96, 95\% Cl $=1.12-3.23 ; P<0.05)($ Table 4).

\section{Discussion}

Despite a lack of research into the potential relationship between cholesteatoma and $\mathrm{DD}$, changes in psychological status have been observed in patients with cholesteatoma [7]. A lack of evidence supporting a link between the two disease entities reflects the priority of surgeons, which leads them to treat the disease according to the rate of recidivism and hearing outcomes $[10,11]$. To the best of our knowledge, this is the first large-scale epidemiologic study to investigate the risk of developing DD following cholesteatoma diagnosis.

In this 10-year cohort study on 10,920 patients, we identified a prospective link between cholesteatoma diagnosis and the development of DD. Compared to those without cholesteatoma, the risk of developing depression increased by 1.90-, 1.51-, and 1.69-times in 1-, 2-, and 3-year follow-up periods, respectively, following adjustment for co-variables (Table

\begin{tabular}{|c|c|c|c|c|c|c|c|c|c|}
\hline \multirow[b]{2}{*}{$\begin{array}{l}\text { Development of } \\
\text { depressive disorders }\end{array}$} & \multicolumn{3}{|c|}{ 1-year follow-up period } & \multicolumn{3}{|c|}{ 2-year follow-up period } & \multicolumn{3}{|c|}{ 3-year follow-up period } \\
\hline & $\begin{array}{l}\text { Patients with } \\
\text { cholesteatoma }\end{array}$ & Comparison & P value & $\begin{array}{l}\text { Patients with } \\
\text { cholesteatoma }\end{array}$ & Comparison & $P$ value & $\begin{array}{l}\text { Patients with } \\
\text { cholesteatoma }\end{array}$ & Comparison & $P$ value \\
\hline Yes (\%) & $23(1.3)$ & $52(0.6)$ & & $35(1.9)$ & $100(1.1)$ & & $53(2.9)$ & $149(1.6)$ & \\
\hline No (\%) & $1797(98.7)$ & $9048(99.4)$ & & $1785(98.1)$ & $9000(98.9)$ & & $1767(97.1)$ & $8951(98.4)$ & \\
\hline Crude HR $(95 \%$ CI) & $\begin{array}{c}2.22 \\
(1.36-3.63)^{\star *}\end{array}$ & 1 & 0.001 & $\begin{array}{c}1.76 \\
(1.20-2.59)^{\star \star}\end{array}$ & 1 & 0.004 & $\begin{array}{c}1.79 \\
(1.31-2.45)^{\star \star \star}\end{array}$ & 1 & $<0.001$ \\
\hline Adjusted HR (95\% Cl) & $\begin{array}{c}1.96 \\
(1.17-3.32)^{\star}\end{array}$ & 1 & 0.011 & $1.51(1.01-2.28)^{*}$ & 1 & 0.047 & $\begin{array}{c}1.69 \\
(1.21-2.36)^{\star \star}\end{array}$ & 1 & 0.002 \\
\hline
\end{tabular}

Total sample number=10920; both crude and adjusted HRs were calculated by Cox proportional hazard regressions, and stratified by age and sex. Adjustments were made for patients' sex age, urbanization level, monthly income, geographic region, hyperlipidemia, and diabetes. ${ }^{*}$ Indicates $p<0.05 ; * \star$ Indicates $p<0.01 ; * \star *$ Indicates $p<0.001$ 
Table 4. Overall and age- and gender-specific incidence densities and relative hazard of depressive disorders in the cholesteatoma group and control group

\begin{tabular}{|c|c|c|c|c|c|c|c|c|}
\hline \multirow[t]{2}{*}{ Variable } & \multicolumn{3}{|c|}{ Case group } & \multicolumn{3}{|c|}{ Control group } & \multirow[t]{2}{*}{$\mathrm{HR}$} & \multirow[t]{2}{*}{ AHR } \\
\hline & $\begin{array}{c}\text { Incident } \\
\text { cases }\end{array}$ & Person-year & $\begin{array}{l}\text { ID per } 1000 \\
\text { patient-years }\end{array}$ & $\begin{array}{c}\text { Incident } \\
\text { cases }\end{array}$ & Person-year & $\begin{array}{l}\text { ID per } 1000 \\
\text { patient-years }\end{array}$ & & \\
\hline \multicolumn{9}{|l|}{ Age } \\
\hline $0-19$ & 1 & 350.67 & $2.85(-2.73-8.43)$ & N/A & 1756.19 & N/A & N/A & N/A \\
\hline $20-39$ & 10 & 1122.95 & $8.91(3.41-14.40)$ & 11 & 5684.73 & $1.94(4.37-7.04)$ & $4.59(1.95-10.82)^{\star \star *}$ & $4.05(1.60-10.24)^{* *}$ \\
\hline $40-59$ & 21 & 2436.46 & $8.62(4.95-12.29)$ & 70 & 12263.28 & $5.71(4.37-7.04)$ & $1.51(0.93-2.46)$ & $1.28(0.76-2.17)$ \\
\hline$\geq 60$ & 21 & 1465.71 & $14.33(8.24-20.41)$ & 68 & 7391.08 & $9.20(7.02-11.38)$ & $1.56(0.96-2.54)$ & $1.57(0.93-2.64)$ \\
\hline \multicolumn{9}{|l|}{ Sex } \\
\hline Female & 32 & 2758.25 & $11.60(7.61-15.60)$ & 100 & 13933.48 & $7.18(5.78-8.58)$ & $1.62(1.09-2.41)^{*}$ & $1.63(1.07-2.48)^{*}$ \\
\hline Male & 21 & 2617.55 & $8.02(4.61-11.44)$ & 49 & 13161.81 & $3.72(2.68-4.76)$ & $2.15(1.29-3.59)^{\star \star}$ & $1.78(1.02-3.10)^{*}$ \\
\hline \multicolumn{9}{|l|}{ Follow up period } \\
\hline Within 1 year & 23 & 1807.97 & $12.72(7.56-17.89)$ & 52 & 9080.64 & $5.73(4.17-7.28)$ & $2.22(1.36-3.63)^{\star \star}$ & $1.96(1.17-3.32)^{*}$ \\
\hline Within 2 years & 35 & 3599.27 & $9.72(6.52-12.93)$ & 100 & 18111.53 & $5.52(4.44-6.60)$ & $1.76(1.20-2.59)^{\star \star}$ & $1.51(1.01-2.28)^{\star}$ \\
\hline Within 3 years & 53 & 5375.79 & $9.86(7.22-12.50)$ & 149 & 27095.29 & $5.50(4.62-6.38)$ & $1.79(1.31-2.45)^{\star \star *}$ & $1.69(1.21-2.36)^{\star \star}$ \\
\hline
\end{tabular}

Total sample number $=10920$; both crude and adjusted HRs were calculated by Cox proportional hazard regressions, and stratified by age and sex. Adjustments were made for patients' sex, age, urbanization level, monthly income, geographic region, hyperlipidemia, and diabetes. N/A, not assessed. * Indicates $p<0.05$; ** Indicates $p<0.01$; *** Indicates $p<0.001$

3). We also determined that the younger cohort (aged 20 to 39 years), male patients, and patients that had been diagnosed within one year had the greatest risk of developing DD (Table 4).

The underlying mechanisms linking depression to cholesteatoma are not yet clear; however, a number of plausible biological and psychosocial factors could play a role. First, invasive cholesteatoma is associated with poor functional prognosis $[12,13]$. Second, cholesteatoma can have profound and far-reaching consequences for daily life, such as the frequent occurrence of foul-smelling otorrhea, hearing loss, tinnitus, vertigo, and even the possibility of facial paralysis or intracranial fatal complications [4]. Patients who are not candidates for surgery (e.g. immunocompromised and the elderly) require frequent visits to the clinic for ear cleaning, which can be highly inconvenient, particularly for busy patients and those who live in rural or remote areas. Patients with problems associated with a post-operative cavity may also face the need for lifelong aural toilet, limitations in their exposure to water, caloric stimulation vertigo when cold air or water enters the cavity, delayed recovery, a cosmetically unpleasing appearance, or difficulties in fitting hearing aids [4]. The threat of recidivism (5-17\% for canal wall down technique; $9-70 \%$ for canal wall up technique) [14] may also raise fears among patients regarding the need for repeated surgeries, which imposes an additional economic burden on patients [4]. Given the aforementioned difficulties, patients may become psychologically inhibited and socially withdrawn, such that DD would be a possible consequence of the physical impairments brought about by cholesteatoma.

Our study found previously unrecognized evidence linking DD to cholesteatoma, and these findings could have strong clinical implications. First, DD has been implicated in poor adherence to medical treatment $[6,9,15]$. Poor follow-up compliance is a major concern of surgeons, even in cases where cholesteatoma has been entirely eradicated, due to the difficulties associated with post-operative recidivism, particularly the need for repeated revision surgeries [16]. Second, research has revealed that depression can increase the morbidity of existing medical conditions $[5,6,17]$. The medical costs of comorbid depression have also been shown to exceed those of medical illness alone. Moreover, functional impairments associated with depression may further increase the economic burden faced by patients $[6,17]$. These issues underline the importance of raising awareness among clinicians with regard to the possibility of comorbid depression in patients suffering from cholesteatoma.

Researchers have demonstrated the significant effects that depression can have on adverse health behaviors (e.g. unhealthy diet, smoking, and sedentary lifestyle) [17], which are now seen as being important risk factors for heart disease, stroke, and other conditions [18]. Functional impairments including occupational disability, reduced productivity, and increased healthcare utilization are common among patients with major depression [17]. The World Health Organization has predicted that depression will continue to be a leading cause of disability, second only to cardiovascular disease by 2020 [19]. A continued increase in disabilities associated with depression implies that many cryptic cases will remain undetected $[5,6]$. Our discovery of a prospective link between cholesteatoma and depression may serve as an early warning sign for potentially unrecognized DD.

One major concern regarding our observations that must be addressed is the true incidence of DD following cholesteatoma diagnosis. In Taiwan, the perceived social stigma of a psychiatric diagnosis often raises fear among patients. Few patients voluntarily report depressive symptoms during physical examinations, and many patients with depression are unwilling to seek psychiatric care. Moreover, some patients fail to meet the diagnosis criteria for clinical depression, despite the presentation of depressive symptoms. Thus, the true incidence of DD following cholesteatoma diagnosis may have been underestimated. This issue warrants further attention.

Depression among children with cholesteatoma should also be given further consideration in future research. The rate of cholesteatoma recidivism among children is reported to be 2 to 10 times higher than among adults, which ultimately leads to a higher incidence of revision surgeries [4]. In addition, hearing loss can have a profound effect on language development, learning, and academic performance. Children who suffer from cholesteatoma are prone to social isolation, which can lead to depression and hinder the development of social skills [11]. Nonetheless, in this study, the incidence density of DD among children with cholesteatoma was only 2.85 per 1000 person-years, which is far lower than incidence rates observed in other age groups (Table 4). This paradoxical observation between children and adults may be explained by the fact that children are more susceptible to social stigma or psychological scarring. Thus, it is highly likely that the true rate of depression among children with cholesteatoma has been underestimated, which suggests the need for routine screening of depressive symptoms among children.

The strength of this study lies primarily in the application of the nationwide NHI dataset. No previous studies have sought to link cholesteatoma to $\mathrm{DD}$, which may be partly due to low cholesteatoma incidence, the small sample size typical of individual institutes, and high rates of individuals lost to follow-up $[11,16,20]$. This current population-based survey provides strong evidence to validate the newly discovered link between 
cholesteatoma and DD thanks to a large sample size, minimal selection bias, and the complete follow-up of cohort members, given that NHI program is a compulsory universal healthcare system. The accuracy of diagnosis in this study is considered acceptable, based on the following: (1) the healthcare system provides complete medical coverage to nearly all residents in Taiwan (99\%) and (2) very low copayment requirements (3 to 15 US dollars) lead to high utilization of medical services, which reduces the possibility of underestimating the number of cases [20]. In addition, patients with otologic symptoms (e.g. otorrhea and hearing loss) are generally referred for further otolaryngologic examination, such that cholesteatoma is theoretically diagnosed or confirmed by an otolaryngologist Furthermore, in Taiwan, only psychiatrists are permitted to make a diagnosis of DD [9].

Despite these strengths, findings in this study should be interpreted with caution. First, an epidemiological association does not necessarily imply biological causation. In this study, we analyzed newly diagnosed cholesteatoma patients to avoid the potential effects of chronicity [9]. It should be noted however, that some of these cases may not have been newly developed. A possible time lag between development and diagnosis may impede estimations about the effects of disease duration on the occurrence of comorbidities. Furthermore, the findings of this study can be generalized to the Taiwanese population; however, applicability to other individuals of other ethnicities requires further validation. Finally, the results obtained in this population-based study require additional confirmation in comparable, longitudinal prospective studies.

\section{Conclusion}

Newly discovered epidemiological evidence linking cholesteatoma to depression suggests that routine screening and monitoring of psychological status among cholesteatoma patients is warranted. Policies aimed at the early detection and timely treatment of comorbid depression following diagnosis with cholesteatoma could enhance health promotion and disease prevention.

\section{Keywords}

Cholesteatoma; depression; hearing loss; population-based study.

\section{Abbreviation}

DD, depressive disorder.

\section{Article Information}

\section{Conflict of Interest Disclosures: None}

Funding: This study was sponsored by grants 10507 and 10626 from Taoyuan Armed Forces General Hospital (Dr Kuo) and grants TMU105AE1-B05 and 106-CGH-TMU-02 from Taipei Medical University (Dr Chang).

\section{References}

1. Semaan MT, Megerian CA. The pathophysiology of cholesteatoma. Otolaryngologic Clinics of North America 2006; 39: 1143-1159.

2. Aquino JE, Cruz Filho NA, de Aquino JN. Epidemiology of middle ear and mastoid cholesteatomas: study of 1146 cases. Brazilian Journal of Otorhinolaryngology 2011; 77: 341-347.

3. Kuo CL. Etiopathogenesis of acquired cholesteatoma: prominent theories and recent advances in biomolecular research. The Laryngoscope 2015; 125: 234-240.

4. Kuo CL, Liao WH, Shiao AS. A review of current progress in acquired cholesteatoma management. European archives of oto-rhino-laryngology : official journal of the European Federation of Oto-Rhino-Laryngological Societies 2014.

5. Benton T, Staab J, Evans DL. Medical co-morbidity in depressive disorders. Annals of clinical psychiatry : official journal of the American Academy of Clinical Psychiatrists 2007; 19: 289-303.

6. Evans DL, Charney DS. Mood disorders and medical illness: a major public health problem. Biological Psychiatry 2003; 54: 177-180.

7. Stayingpositive: Suffering depression prior to onset/diagnosis of cholesteatoma. In: Cholesteatomanet Message Boards 2014.

8. Wu PH, Lin YT, Kuo CN, Chang WC, Chang WP. No increased risk of herpes zoster found in cirrhotic patients: a nationwide populationbased study in Taiwan. PloS One 2014; 9: e93443.

9. Chen $\mathrm{YH}$, Keller JK, Kang JH, Hsieh HJ, Lin HC. Obstructive sleep apnea and the subsequent risk of DD: a population-based follow-up study. Journal of clinical sleep medicine : JCSM : official publication of the American Academy of Sleep Medicine 2013; 9: 417-423.

10. Jung KH, Cho YS, Hong SH, Chung WH, Lee GJ, Hong SD. Quality-of-life assessment after primary and revision ear surgery using the chronic ear survey. Archives of Otolaryngology--Head \& Neck Surgery 2010; 136: 358-365.

11. Kuo CL, Shiao AS, Liao WH, Ho CY, Lien CF. Can long-term hearing preservation be expected in children following cholesteatoma surgery? Results from a 14-year-long study of atticotomy-limited mastoidectomy with cartilage reconstruction. Audiology \& Neuro-Otology 2012; 17: 386-394.

12. Lailach S, Kemper M, Lasurashvili N, Beleites T, Zahnert T, Neudert M. Health-related quality of life measurement after cholesteatoma surgery: comparison of three different surgical techniques. European archives of oto-rhino-laryngology : official journal of the European Federation of Oto-Rhino-Laryngological Societies 2015; 272: 3177-3185.

13. Quaranta N, Iannuzzi L, Petrone P, D'Elia A, Quaranta A. Quality of life after cholesteatoma surgery: intact-canal wall tympanoplasty versus canal wall-down tympanoplasty with mastoid obliteration. The Annals of Otology, Rhinology, and Laryngology 2014; 123: 89-93.

14. Tomlin J, Chang D, McCutcheon B, Harris J. Surgical technique and recurrence in cholesteatoma: a meta-analysis. Audiology \& Neuro-Otology 2013; 18: 135-142.

15. Evans DL, Charney DS, Lewis L, et al. Mood disorders in the medically ill: scientific review and recommendations. Biological Psychiatry 2005; 58: 175-189.

16. Kuo CL, Shiao AS, Liao WH, Ho CY, Lien CF. How long is long enough to follow up children after cholesteatoma surgery? A 29-year study. The Laryngoscope 2012; 122: 2568-2573.

17. Katon WJ. Clinical and health services relationships between major depression, depressive symptoms, and general medical illness. Biological Psychiatry 2003; 54: 216-226.

18. Boden-Albala B, Southwick L, Carman H. Dietary interventions to lower the risk of stroke. Current Neurology and Neuroscience Reports 2015; 15: 15.

19. Michaud CM, Murray CJ, Bloom BR. Burden of disease--implications for future research. JAMA 2001; 285: 535-539.

20. Kuo CL, Lien CF, Shiao AS. Mastoid obliteration for pediatric suppurative cholesteatoma: long-term safety and sustained effectiveness after 30 years' experience with cartilage obliteration. Audiology \& Neuro-Otology 2014; 19: 358-369. 